\title{
Active surveillance of localized disease offers improved survival in the PSA era
}

Survival rates in patients with localized prostate cancer managed with watchful waiting are significantly better than they were before the advent of PSA screening, according to the results of a large US population-based study. "Our most significant finding is that the 10 -year risk of dying from prostate cancer is reduced by more than $60 \%$ among the majority of patients in our cohort compared with men diagnosed in the 1970s and 1980s," claims Grace Lu-Yao, co-author of the study.

Lu-Yao and colleagues used data from the merged Medicare and SEER databases to identify 4,455 men aged $65-74$ years who were diagnosed with clinically localized (T1 or $\mathrm{T} 2$ ) prostate cancer between 1992 and 2002, and did not undergo surgery or radiation therapy in the 6 months following initial diagnosis. They compared their results with the outcomes of patients diagnosed prior to 1988-before PSA screening was widely used-from the available literature.

\section{10-year disease-specific} survival was $94 \%$ with watchful waiting, a figure difficult to improve upon 77

10 years after diagnosis, only $2-6 \%$ of men with moderately differentiated disease (Gleason grade 5-7) had died from prostate cancer in the contemporary study, compared with $15-23 \%$ in the pre-PSA period. In other words, 10 -year disease-specific survival was $94 \%$ with watchful waiting, a figure difficult to improve upon. "We cannot assume that cancer interventions will improve survival or quality of life," says Lu-Yao. "For many patients with low-risk cancer, the side effects of treatment might outweigh its potential benefits."

Such high survival rates suggest conservative management is a feasible treatment choice for older men with localized disease, an idea supported by the recent finding of the Scandinavian Prostate Cancer Study Group that prostatectomy offers no survival benefit for patients over the age of 65 years.

The investigators intend to continue their study in order to obtain data from longer follow-up. They are also planning to evaluate the influence of radiation therapy on survival rates in various risk groups.

Sarah Payton

Original article Lu-Yao, G. L. et al. Outcomes of localized prostate cancer following conservative management. JAMA 302, 1202-1209 (2009). 